\title{
From Hierarchical Control to Flexible Interactive Electricity Services: A Path to Decarbonization
}

\author{
Marija D. Ilić \\ MIT Cambridge, MA 02139 USA \\ ilic@mit.edu
}

\author{
Pedro M. S. Carvalho \\ IST, Lisbon, Portugal \\ pcarvalho@tecnico.ulisboa.pt
}

Received: April 18, 2021. Received: September 7, 2021. Accepted: October 7, 2021. Published: October 11, 2021.

\begin{abstract}
We propose to conceptualise electric energy systems as complex dynamical systems using physically intuitive multilayered energy modelling as the basis for systematic diverse technology integration, and control in on-line operations. It is shown that such modelling exhibits unique structure which comes from the conservation of instantaneous power $(P)$ and of instantaneous reactive power $(\dot{Q})$, (interaction variables (intVar)) at the interfaces of subsystems. The intVars are used as a means to model and control the interactive zoomed-out inter-modular (inter-area, inter-component) system dynamics. Control co-design can then be pursued using these models so that the primary control shapes intVars of its own module by using its own lowlevel detailed technology-specific model and intVar info exchange with the neighbours. As a result, we describe how the proposed approach can be used to support orderly evolution from today's hierarchical control to a platform enabling flexible interactive protocols for electricity services.

The potential for practical use of the proposed concepts is far-reaching and transparent. All that needs to be conceived is that intVar characterising any intelligent Balancing Authority (iBA) is a generalisation of today's Area Control Error (ACE) characterising net energy balance of a Balancing Authority (BA). An iBA can be any subsystem with its own sub-objectives, such as distributed energy resources (DERs) comprising customers and grid forming microgrids; distribution systems; transmission systems; Independent System Operators (ISOs); and, ultimately, electric energy markets within large interconnection. Several industry problems are described as particular sub-problems of general interactive electricity services. These formulations help one compare models and assumptions used as part of current solutions, and propose enhanced solutions. Most generally, feasibility and stability conditions can be introduced for ensuring feasible power flow solutions, regulated frequency and voltage and orderly power exchange across the iBAs.
\end{abstract}

Index Terms-Hierarchical electric power systems with intermittent resources; Supervisory Control and Data Acquisition (SCADA); Scalable multi-layered modelling and control of energy dynamics; Inter-area dynamics and control; Distributed interactive electricity service.

\section{INTRODUCTION}

This paper concerns modelling and control of electric energy systems comprising diverse technologies embedded within several voltage levels of a complex electric power grid, Figure 1. The main question concerns theoretical and practical enhancements needed to provide reliable, efficient and sustainable electricity service. Similarly, when designing new stand-alone microgrid architectures, it is important to have quantifiable and reproducible methods for both justifying specific technology deployment and for its best on-line utilisation. Major regulatory initiatives mandate the integration of distributed energy resources (DERs) and their utilization in a flexible and efficient way. Proposed flexibility markets call for new products/services, such as balancing, congestion management, voltage control, islanding, and restoration support. Implementation of these highly heterogenous products across different ownership boundaries, often referred to in the US as "seams", will require end-to-end integration according to welldefined protocols/standards over both horizontal (cross-border) and vertical (across ISOs, TSOs, DSOs) temporal, spatial, functional boundaries and flexible multi-stage utilization of resources and demand management.

In this paper we suggest that supporting these changes in system operations will require to further conceptualize new electric power system architectures which are fundamentally different from today's tertiary, secondary and primary level top-down EHV/HV hierarchical architectures. The emerging architectures are generally nested, multi-layered and comprise multiple heterogeneous decision makers with highly diverse technical, economic and societal sub-objectives. New modelling, decision tools and software applications are needed at different system layers to support the interactions over time and geography. There exists a general mismatch between this newly emerging need and the assumptions underlying today's hierarchical control. We summarize these assumptions and explain why they are no longer valid. In particular, models and methods based on real-reactive power decoupling, linearization around pre-specified scenarios and pre-programmed/constantgain PID control embedded in primary level become major roadblocks to operating in a flexible way over broad ranges of conditions. In short, today's hierarchical control is: static, deterministic; focused on central EHV/HV/MV grid control; requires large preventive reserves; does not rely much on real time corrective, flexible, actions; finally, it does not enable systematic participation of MV/LV/DERs. This results in: significant waste through excess reserves (typically 20$30 \%$ unused reserves); significant waste due inefficient use of existing infrastructure (only $30 \%$ of transmission capacity currently used); very costly long distance transmission, and poor resilience (e.g. California); limited renewables capacity share that can be accommodated (In Puerto Rico, system claims that $15 \%$ is max, our simulations show that much higher transmission capacity utilization is possible with no change in physical transmission system) [3]; lack of resilience to major storms, failures, attacks [4]; and, lack of ability for communities and other stakeholders to "push the envelope" 
on environmental impact, efficiency without major sacrifices in scale/pooling efficiency. In contrast, efficient energy service requires temporal, spatial and functional alignment of energy resources and demand; and, T\&D system needs to be operated to integrate the growing number of DERs, storage and intermittent resources in a flexible data-enabled way in order to manage uncertainties in an efficient manner.

In this paper we address the fundamentals of modeling and control needed to support innovations in changing industry. To that end, we first briefly review today's bulk power system (BPS) hierarchical modelling and control and the supporting SCADA in Section II. We stress the implied assumptions which form fundamental roadblocks to operating the emerging systems in sustainable way on the road to decarbonization. In Section III we introduce a new multi-layered energy modelling in support of interactive end-to-end electricity services which no longer requires these assumptions. Instead of modelling and controlling the complex system as a centralized top down BPS, the modelling is modular and interactive. The key theoretical result is that the grid-connected entities with their own sub-objectives, utility-or non-utility owned, are modelled in terms of common, technology-agnostic interaction variables(intVars). Starting from first principles, it can be shown that these variables have unique properties which make it possible to re-integrate seemingly balkanized grid stakeholders so that feasible and stable operations can be implemented. Notably, in addition to be mathematically sound, they have a straightforward physical interpretation as instantaneous power, rate of change of instantaneous reactive power and energy. As a result, the interactions between different layers of the complex electrically-interconnected grid, secondary and tertiary, can be understood by assessing power and energy dynamics of interconnected subsystems, and are technology agnostic. In Section IV we describe today's hierarchical model as a particular example of this more general model. We discuss three major industry problems, and summarise upto-date findings and enhanced solutions which are effectively examples of multi-layered energy modelling and control usage. Finally, in the closing Section V we propose further work toward systematic protocols founded on minimally coordinated distributed interactive multi-lateral information exchange in support of reliable and efficient innovation at value.

\section{TODAY'S BPS AND SCADA}

Shown in Figure 2 is a sketch on Supervisory Control and Data Acquisition (SCADA)-enabled information processing in today's Bulk Power Systems (BPS) [2]. Blue dotted lines represent the information exchange between the BPS and the substation. Shown in Figure 3 are the corresponding functional hierarchies [5]. While not fully standardised and somewhat system-specific, hierarchical control of each BPS generally comprises three levels of data processing and control. A closer look into state-of-the-art and computer applications in today's hierarchical control reveals qualitatively different models used for different levels of hierarchy. These models have been introduced and improved over time, but the assumptions regarding the inter-dependence with the other layers have generally remained. Most generally, BPS tertiary-level models used in computer applications by their Energy Management Systems (EMS) are most standardised and based on steadystate assumptions, for example in power flow analysis, Security Constrained Economic Dispatch (SCED) and Unit Commitment (UC). The small signal and transient stability analyses are performed off-line for the set of selected scenarios. Only steady state models are used in on-line feed-forward computer applications.

\section{A. Tertiary level modelling and control}

Tertiary level is the EMS for the entire system, could manage several Balancing Authorities (BAs), Figure 4 shows the complexity of the Northeast Power Coordinating Council (NPCC) area [6]. Figure 5 shows part of the European Union interconnection. Tertiary level performs SCED and UC in support of real power dispatch so that predictable system load obtained using SCADA measurements on power plants and BPS level substations is supplied. It also sets energy exchange agreements with the neighbouring systems.

Need for optimal voltage dispatch: Voltage dispatch is not standardised and since (Independent) System Operator ((I)SO) does not own nor operate voltage controlled equipment voltage dispatch is performed by the Transmission System Operators (TSOs) in their own satellite control centres. The performance objectives for voltage dispatch are not fully specified by the regulatory bodies. Frequently more advanced TSOs optimise their voltage profile to minimise delivery losses to enhance physical efficient power delivery. Notably, that voltage profile is not most optimal for maximising economic efficiency and social welfare, which are the main objectives of the (I)SOs and electricity markets. This, in turn, further leads to a lack of explicit incentives for optimising voltage profile, a wellrecognised issue [7].

Conservative interconnection-level dispatch (cross-border, seams operating problems): Utilities are required to compute and post their Available Transfer Capability (ATC) for enabling open non-discriminatory access to their grid when power is generated and delivered within the interconnection. Shown in Figure 6 is a sketch of three interconnected BAs, each having its own (I)SO. At present each BA schedules its own resources to supply predicted load in its own area, and according to energy exchange agreements with the neighbouring BAs. The ATC is typically computed in an entirely decentralised manner, without any coordination of tie-line flow exchanges. This approach is conservative because the ATC of the interconnected system is generally much higher than the ATC computed in a decentralised way. Notably, if ATC is computed using DC OPF by each ISO/electricity market, the interconnected system with the targeted tie line flows may not be feasible. In particular, there are major cross-area problems with voltage/reactive power management. Notably, lack of incentives to support optimal system-level voltage leads to failures to optimise welfare and performance of electricity markets, and ultimately results in reduced ability to pursue 


\section{HV SYSTEM}

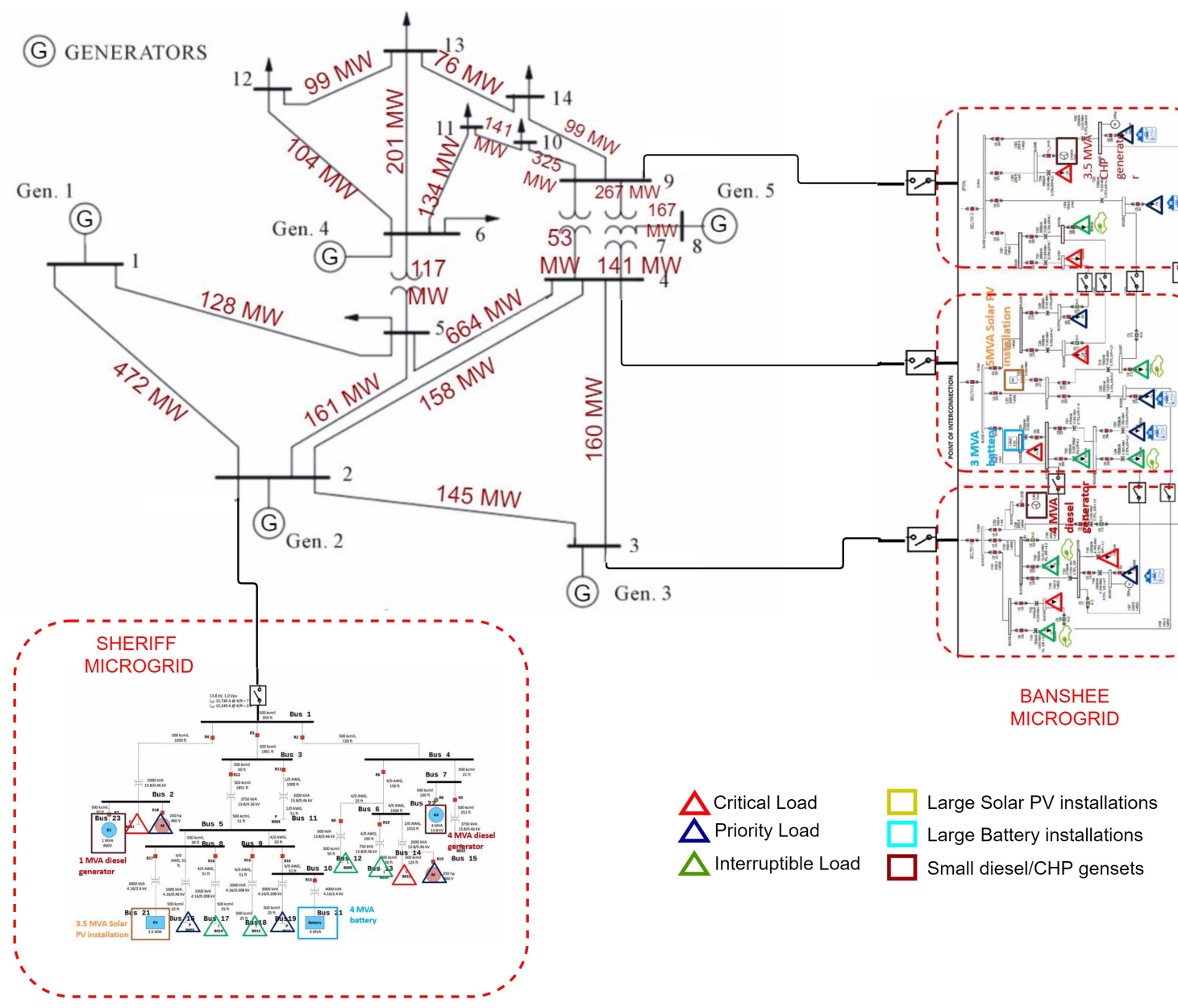

Fig. 1. Emerging end-to-end interacting electric energy systems; different subsystems form interacting iBAs [1]

decarbonisation through the integration of on-shore and offshore clean resources and the delivery of their power to the right locations. Voltage-related constraints are typically the first binding constraint to such delivery; documented is, for example, that the US Northeastern Power Coordinating Council (NPCC) interconnected system could deliver $>1 G W$ more hydro power from Niagara to NYC on a hot summer day than it is currently done if voltage profile were optimised [8], [10], [21]. Figure 6 shows qualitatively different exchanges between the BAs depending on how is interconnection-level dispatch done. It can be seen that the NY-NE tie-line energy exchange can be much higher when voltage is optimally dispatched. These benefits may appear counter-intuitive because of localised voltage response property [12]. To understand the effects of a robust AC OPF when optimising both voltage and real power it is important to understand that when voltage is not treated as a constraint but a controllable decision variable, the most critical constraints are found and something else is adjusted so that they are no longer active. In simple words, voltage adjustment at the right place makes the power delivery physically implementable.

Intra-area dispatch problems: Recall from Figure 1 that even within a single BA there exist interactions between transmission, distribution and end-users levels. The dynamics of distribution systems are generally not modelled for purposes of control and protection. Instead, control and protection settings of very large number of controllable distribution equipment, capacitor banks and transformers, are pre-programmed assuming top-down power flows. Short circuit analyses are done at the planning stage to ensure that protection settings would make the grid safe during faults. Probably it was Judith Cardell who in her early work was the first one to draw attention to possible frequency insatiability problems in distribution systems with many DERs [13], [14]. Most recently, connecting many DERs into distribution grids has led to concerns regarding their effects on unacceptable voltage profiles, particularly in systems with many solar resources and price-responsive demand [15]. Another complexity in attempting to deliver most power is current requirement for substations to maintain nearunity power factor of the areas they serve. This requirement 


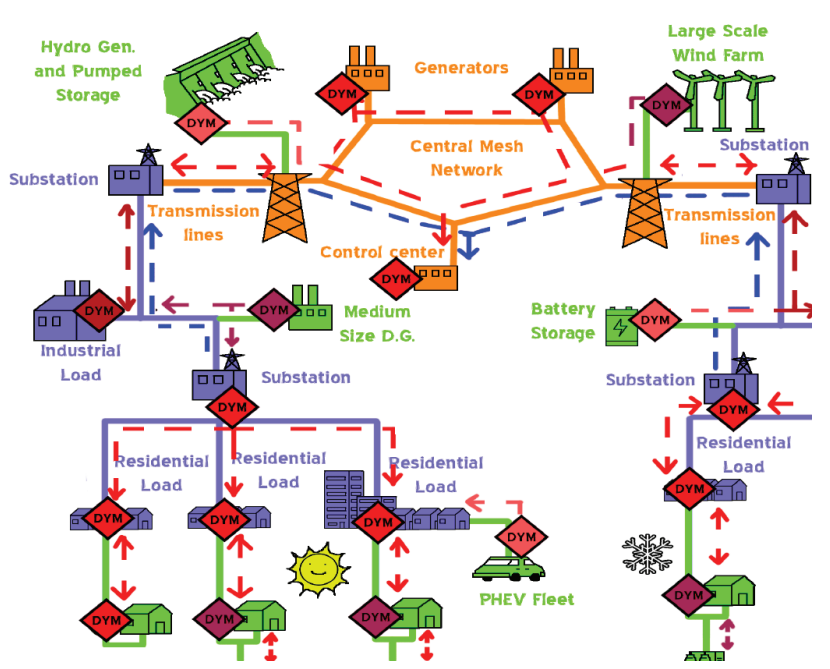

Fig. 2. Model-based informational protocols in future SCADA [2]

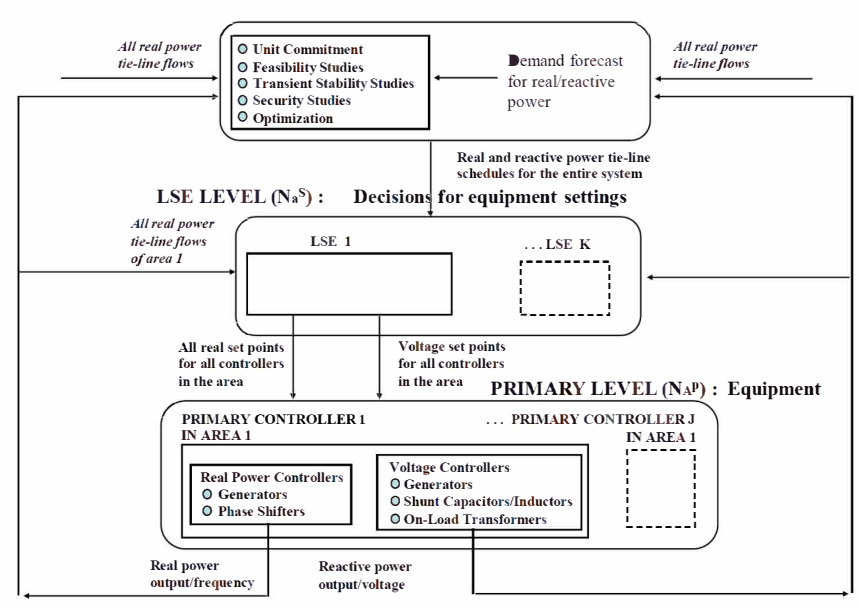

Fig. 3. Functional hierarchies in today's industry [5]

often causes un-necessary wear-and-tear of distribution grid equipment and it does not support the most optimal voltage needed for the BPS to meet its objectives. We also recall that the results obtained by the tertiary level are implemented by sending these to the power plants to adjust the set points of their controllers. The substation level loads are modelled as constant real-reactive power "PQ" loads and generators are modelled as real power-voltage "PV" components. They are subject to ramp rate and capacity limits. Important for the objectives of this paper is to recall that today's hierarchical controllers attempt to make power plaints behave as "PV" components by changing set points of governors and automatic voltage regulators (AVRs). More generally, models used for computer applications at different levels of the hierarchy and for supporting data-enabled decisions and control, have been derived for each specific layer under several assumptions concerning their interactions with other layers and also the models for their own computer applications, summarised next.

\section{B. Secondary-level modelling and control}

Secondary level control is performed by each BA with basic objectives of frequency and voltage regulation in response to relatively slow, minute-rate deviations from predicted system load, commonly known as the Automatic Generation Control (AGC). In some parts of the world Automatic Secondary Level Voltage Control (ASVC) is also implemented by the BAs to regulate so-called " "pilot point" voltages in response to hardto-predict reactive power deviations from those assumed when tertiary level control is done. Important questions concerning selection of pilot points and potential for using PMUs for implementing enhanced ASVC have been recently studied [16], [17].

It is assumed when modelling tertiary level for scheduling that a power plant can control its governor set point $\omega_{G}^{r e f}$ so that real power generation schedule given to the plant $P_{G}^{r e f}$ is implementable. To the best of our knowledge, all computer applications used by the tertiary level for scheduling are written under this assumption. However, for the generator to produce specified $P_{G}^{r e f}$ in a provable way, it is necessary to: i) have its primary control stabilise local output variables to frequency $\omega_{G}^{r e f}$ and voltage magnitude $V_{G}^{r e f}$; and, ii) have a uniquely defined relation between $P_{G}$ and these set points that are actually controlled ("knobs turned"). However, the wellknown routinely used decoupled real power-frequency droop

$$
\Delta P_{G}=B \Delta \omega_{G}+M \Delta \omega_{G}^{r e f}
$$

is derived under several assumptions, including i) decoupling of frequency and voltage dynamics; and ii) the G-T-G closed loop dynamics linearisation around the selected operating conditions are valid assumptions. Observe further that this threeway relationship between increments in power generated, local frequency and frequency set point can further be expressed as a two-way relationship assuming increment in actual system frequency is zero, namely the system equilibrium frequency is nominal. Such two way relation that enables adjustments of the set point so that the specified power is generated. If any of these are not valid, namely decoupling, linearization, equilibrium frequency nominal, the generator cannot produce specified power.

Important for purposes of this paper is to point out that while in the past these assumptions have been valid most of the time valid, the emerging changes in operations with intermittent resources bring all of these into question. In particular, it is no longer possible to decouple frequency and voltage dynamics because, fundamentally, fast variations in power cause also significant variations in voltage, and vice versa [19]. Similarly, most of the component models are nonlinear and their dynamics are operating conditions dependent; large deviations in system inputs caused by the intermittent resources no longer make it possible to use linearized models for assumed nominal inputs. Consequently, there is no guarantee that primary controllers would stabilise fluctuations of local frequency and voltages to the specified set points. Consequently, because of variety of these causes, it has been quite well documented 


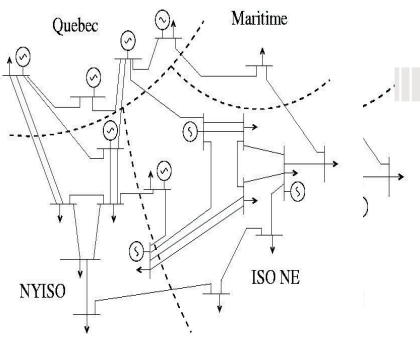

a)

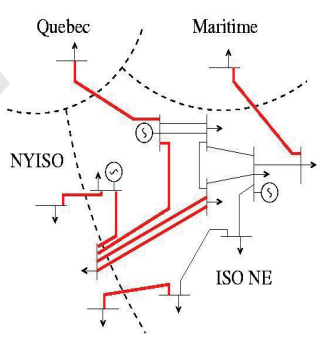

b)

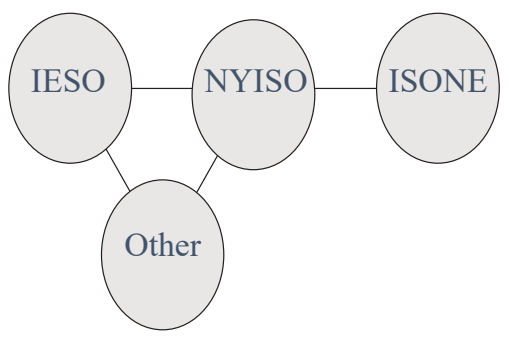

c)

Fig. 4. a) Interconnected system; b) Distributed subsystems; c) Multi-layered interactions

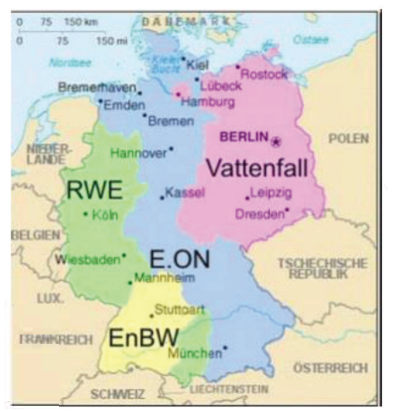

89.3

32.1

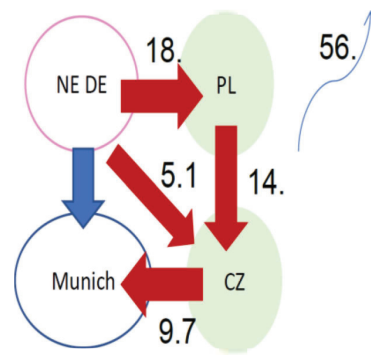

Fig. 5. ATC assessment in Europe: With voltage/reactive power optimisation $30 \mathrm{GW}$ generated $23 \mathrm{GW}$ delivered; Voltage constrained- generated $89.3 \mathrm{GW}$ and $32.1 \mathrm{GW}$ delivered [9]

that system frequency typically deviates from nominal in a persistent way [20].

\section{Primary components modelling and control}

For the secondary level models to be valid, it is implied that physical primary controllers in conventional power plants are tuned to stabilize frequency and voltage errors. Primary level controllers are embedded locally within power plants and other controlled equipment to stabilise local frequency and voltage to the reference values. Conventionally, governors have been tuned to stabilise local frequency and voltage and power system stabilisers (PSSs) to stabilise local voltage. With the deployment of intermittent resources and dispersed fast storage, as well as with enhancements of wire control technologies (FACTS, HVDC, smart wires), the diverse inverter-controlled equipment is contributing in significant ways to fast transient dynamics. Many newly emerging fast instability problems such as control induced system stability (CISS) [21], sub synchronous resonance (SSR) [22], unacceptable transients and inter-area oscillations are currently limiting integration and utilisation of intermittent resources [23]. Because of this, there exists a major need to have systematic well-designed primarylevel control that "works", discussed in some detail later in Section IV. Identifying these emerging fast stability problems
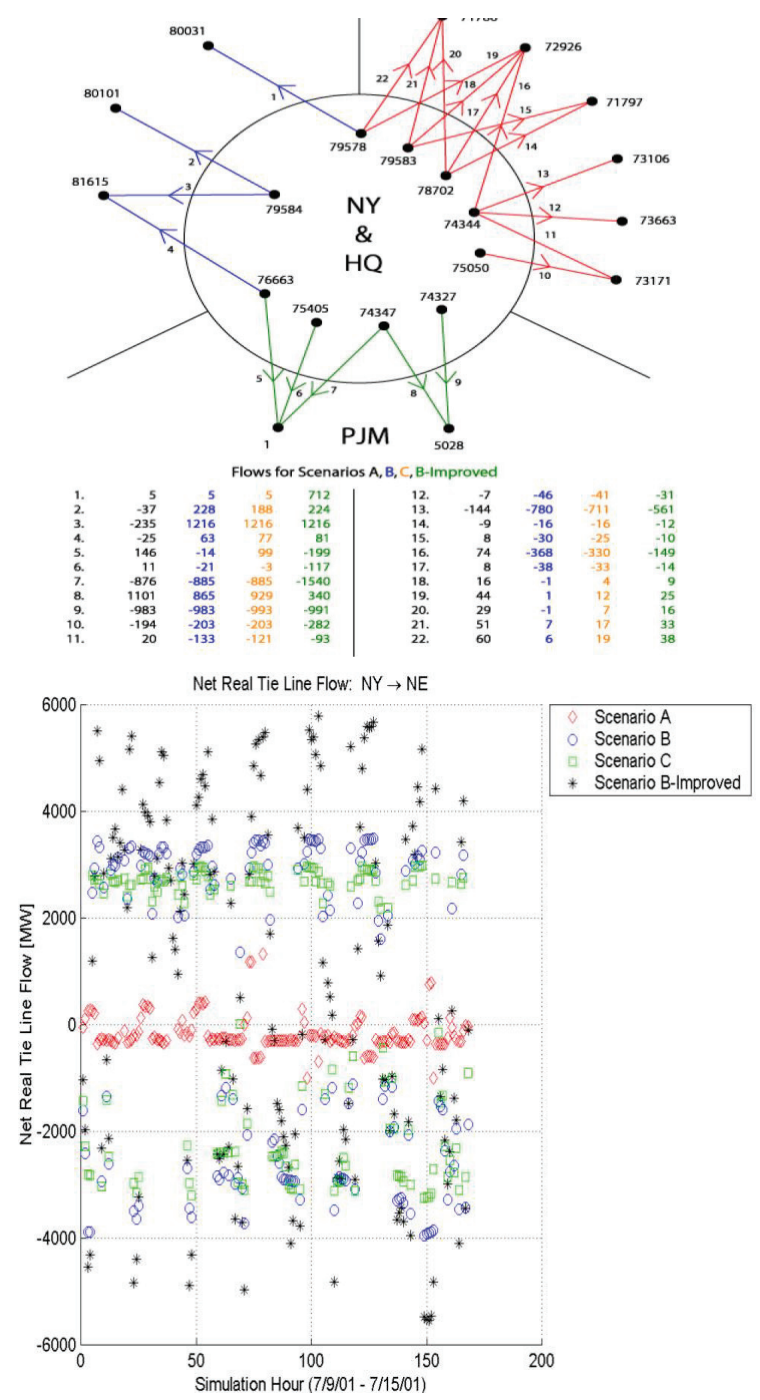

Fig. 6. ATC assessment in NPCC: With voltage/reactive power optimisation (scenario B) NY-NE power exchanges much higher than without (scenario A) [8] 
and revisiting fundamentals for their control are some of the major reasons for writing this paper.

Finally, we point out that both control and protection in distribution grids served by the substations are currently preprogrammed and there is generally no on-line monitoring for purposes of data-enabled feed-forward and feedback adjustments to the ever-changing operating conditions. To fill this gap, there has been recent effort toward designing and deploying Distributed Energy Resource Management Systems (DERMS) and Advanced Demand Management Systems (ADMS). Their expected performance objectives are to enable on-line integration of highly-varying demand and of DERs. Shown in Figure 2 red dotted lines represent an end-toend enhanced multi-layered SCADA under consideration for enabling DERMS/ADMS functions [1]. The scaling up and aggregation challenge of a typically very large number of very small distribution grid users when one attempts to account for their participation in BPS services, such as grid congestion management, remain major challenges [24]. Automatic Generation Demand Control (AGDC) is an example of the basic idea that many very small MV/LV grid users can participate in ancillary services [26], [25] and even in transactive energy management (TEM) [15]. As these opportunities are being explored an open question concerns the overall Information Communications Technology (ICT) architecture design for implementing such end-to-end integration. We point out that instead of implementing scaled-down BPS hub-and-spokelike SCADA, many novel solutions could and should be considered. For example, multi-lateral information exchanges between many small users and their coordinators communicating to the higher-level ICT is a more effective architecture than requiring all, even the smallest users, to be metered and communicated directly with the substation coordinator. Ultimately, it becomes possible to have large portions of self-adapting end users subject to minimal coordination [27]. For this to work, it is necessary to asses fundamental trade off between control, monitoring and communications requirements, on one side, and the achievable performance objectives, on the other side. To do so, we describe modelling of hierarchical sub-processes and their inter-dependencies next.

Ensuring controlled voltage $V_{G}^{r e f}$ is even more problematic, and this is discussed in some detail in Section IV. Fundamentally, balancing continuously time varying power typical of intermittent resources, cannot be done by means of controllers which were tuned against static Thevenin equivalent representation of the system to which the generator, or any other controllable component, are connected; this has been practice in today's industry and, as a result, there has been increasing presence of inter-components, or inter-area, oscillations caused by the lack of ability to control them [28]. This is further illustrated in Section IV, and new controller design is proposed which overcomes this fundamental problem. In practical terms, this problem is manifested, for example, by wind-power plant oscillating against a static var compensator (SVC) or against FACTS controlled wire flow [21].

Modelling assumptions made today in SCED/UC computer applications used in a BPS become even more complex when attempting to represent loads, DERs, and controllable wires. For example, modelling substation as a given PQ load implies that a complex mix of diverse small consumers served by the substation seen by the BPS at the point of connection (POC) to substation has a highly predictable real and reactive power consumption. To begin to assess the validity of this assumption, recall that it is quite difficult to account for feedback effects of price-responsive load on the actual load even at the slowest tertiary level time scale [15]. The secondary and primary level modelling of the effects of diverse very large number of grid-edge physical component dynamics at the time scales relevant for regulation and stabilisation has never been attempted at the large-scale seen in real-world power girds. This task is not implementable by the BPS for variety of reasons, including lack of knowledge of those physical components, their models and parameters. This is in addition to not having computationally feasible methods for their aggregation. Some progress has been made toward modelling microgrids comprising homogeneous inverter-controlled solar rooftops and batteries, particularly for studying grid forming problems of stand-alone microgrids [29], [30], [31]. Scaling up effects of systems of small microgrids is effectively an unsolvable problem as currently attempted. The dynamic effects of highly heterogeneous diverse gird-edge components are therefore practically impossible to capture [32], [33]. On top of these issues, the dynamics of today's distribution grids to which these DERs and microgrids are connected are not modelled.

In short, the key challenge that emerges is how to represent the highly heterogeneous multi-scale, multi-spatial interconnections in a unified, scalable and transparent way with the objective to control undesired dynamical and feasibility problems. The rest of this paper introduces one such modelling approach.

\section{NEW MULTI-LAYERED ENERGY MODELLING}

Instead of making a priori modelling assumptions for technology-specific equipment, we introduce a multi-layered modelling in energy space. The fundamentals of such multilayered approach were introduced some time ago by Willems who argued that it is impossible to accurately capture dynamics of the interconnected physical system by pre-defining input-output characteristics of sub-systems independently from accounting for their dynamic interactions with the system to which they are connected [34]. He further suggested that a more complete sub-system model must comprise its detailed technology-specific variables, he calls latent variables, as well as the shared variables that reflect its dynamical interactions with the other neighbouring components. This far-reaching and somewhat under-utilised general concept proposed for modelling physical systems is basically such that one must start with the given candidate interconnected system, perform tearing (defining boundaries of subsystems), zooming in to define technology-specific complete models of sub-systems 

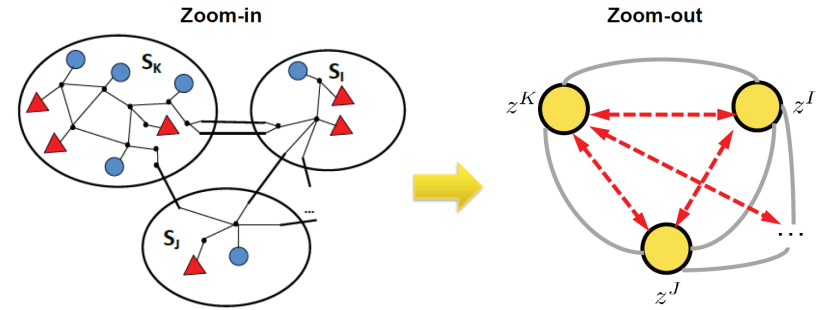

Fig. 7. Zoomed-out representation for the interconnected system [42]

and then combining them into an interconnected system model, conceptualised in Figure 7.

This general idea is not foreign to the power engineering community since tearing methods underlying diakoptics were introduced early on for representing complex power flow networks [36], [35].It is probably worthwhile to recall Inadvertent ZEnergy Exchange (IEE) concept and also interpret it it in terms of interactions between BAs [37]. The new challenge is how to do this in dynamical systems such as electric energy systems comprising temporal, spatial and functional diversities. This generalisation requires conceptualising electric energy systems as complex dynamical systems first. In what follows we first do this and highlight their unique structure which comes from the conservation of instantaneous power and conservation of instantaneous reactive power at the interfaces of subsystems. It then becomes possible to interpret particular models used in today's analysis and control of electric power systems as examples of this general modelling, as illustrated in Section IV. In the remainder of this section

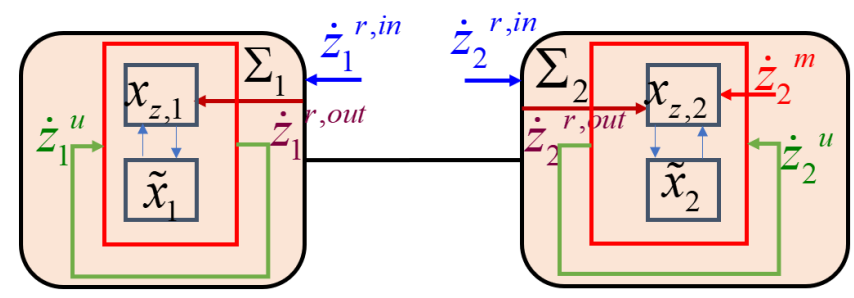

Fig. 8. Zoomed-out representation for the interconnected system [42]

we make the case that the multi-layered structure in electric energy systems is technology-agnostic because there exists a natural choice of shared variables which is based on universal conservation laws. This key observation sets the basis for general multi-layered modelling of any electric energy system, and the theoretical justification for functionality requirements in terms of shared interaction variables. These ideas have emerged over quite some time in our group and only summary of concepts of direct relevance for enabling cooperative electricity service is given here.

\section{A. Definition of interaction variable and its structural prop- erties}

The proposed multi-layered modelling relies on harnessing the unique structural properties of the interaction variable $z_{i}^{r, \text { out }}$ used to characterise interface of module $i$ with the rest of the system [38], [39]. In particular, it can be shown to depend only on its internal state variables; and, it satisfies the property.

$$
z_{i}^{r, \text { out }}=\text { constant }
$$

when all interconnections are removed. Let $E_{i}, p_{i}, P_{i}^{u}$, $P_{i}^{m}, \dot{Q}_{i}^{u}, \dot{Q}_{i}^{m}$ represent the stored energy, rate of change of stored energy, instantaneous power at control terminal and disturbance terminal and generalised rate of reactive power [40], [41] at control and disturbance terminals, respectively, of component $i$. Each of these variables is shown to be computable as a function of local state variables and state derivatives [42]. Mathematically, the interaction variable is then defined as

$$
z_{i}^{r, \text { out }}=\left[\begin{array}{l}
\int_{0}^{t}\left(p_{i}(s)+\frac{E_{i}(s)}{\tau_{i}}-P_{i}^{u}(s)-P_{i}^{m}(s)\right) d s \\
\int_{0}^{t}\left(-\dot{p}_{i}(s)+4 E_{t, i}(s)-\dot{Q}_{i}^{u}(s)-\dot{Q}_{i}^{m}(s)\right) d s
\end{array}\right]
$$

Here $\tau_{i}$ stands for damping reflecting the rate at which stored energy dissipates as Joule thermal losses [39]. In order to differentiate the interactions resulting from internal energy conversion processes, and the ones obtained as a result of interconnection, we utilize the superscripts 'out' and 'in', respectively. The incoming interaction variable is a result of interconnection, as shown in the zoomed-out representation of the interconnected system in Fig. 9. Notice that the outgoing interaction variables as per definition depend only on local state variables and their derivatives. Its time derivative however depends on the rate of change of the incoming interaction variables $\dot{Z}_{i}^{r, i n}$ that are function of state variables of other components in the system.

\section{B. A tearing approach to representing an interconnected elec- tric energy system}

Consider next without loss of generality an end-to-end representative future electric energy system shown in Figure 1. Any such system can be thought of as comprising subsystems (dotted lines) with their own internal states, Figure 7 and for the system comprising two subsystems in Figure 8. The basic tearing defines junctions which divide subsystems, $\Sigma_{1}$ and $\Sigma_{2}$. We point out that tearing is non-unique and can be based on organizational divisions, aggregation of physical components with similar functionalities, portions of subsystems with similar sub-objectives, etc. Important for multi-layered modelling is to think in terms of interaction, shared variables $z_{i}$, between components and their dynamics, as shown in Figure 8.

\section{A zoom-in modelling of subsystems' functionalities using unified technology-agnostic intVars}

Shown in Figure 9 is a sketch of the general structure of subsystem modelling, independent of specific technology. Internal to the subsystem is its model of physical state space dynamics in terms of its own extended state variables $\tilde{x}$, port variables $r$, local disturbances $m$ and local primary control $u$. 


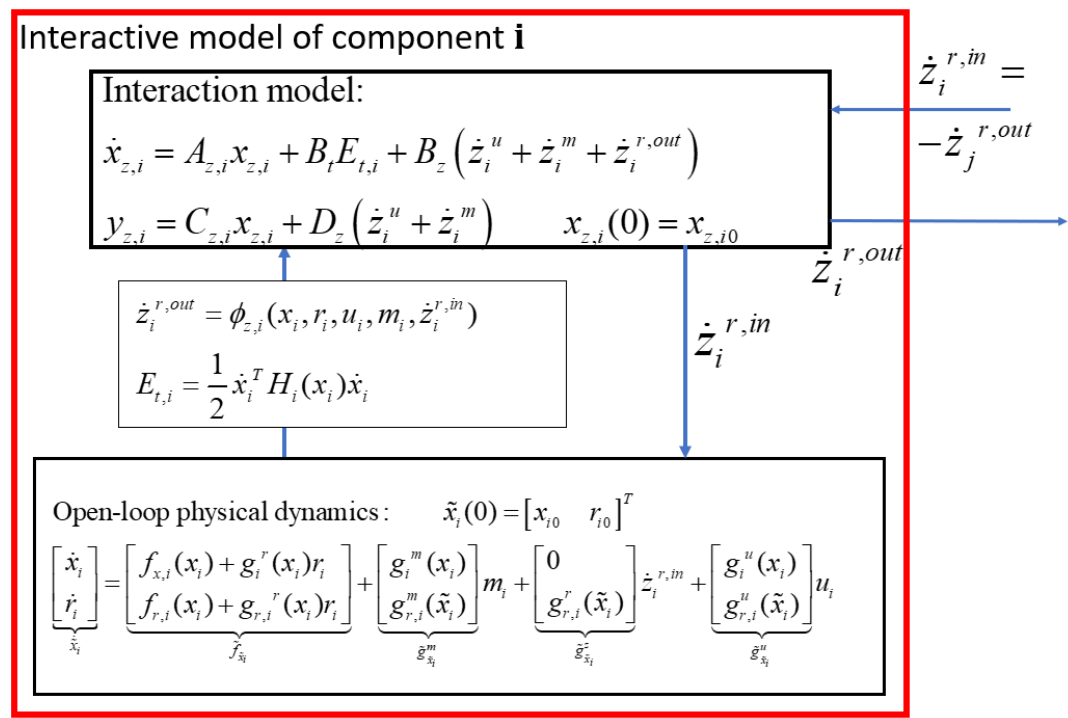

Fig. 9. Interactive stand-alone model of a closed-loop component $i$ : The lower layer models are utilized to compute the outgoing interaction variables $\dot{z}_{i}^{r \text {,out }}$, which drive the higher-layer energy dynamics of the component. The incoming interaction variables from the grid $\dot{z}_{i}^{r \text {,in }}$, are utilized by the lower-layer models to evaluate the extended state trajectories $\tilde{x}_{i}=\left[x_{i}, r_{i}\right]^{T}$ given their initial conditions. The incoming interactions are a result of outgoing interactions of neighbours.

It has been shown that dynamics of each zoomed in subsystem $\dot{x}$ depends on its own state variables $x$, port variables $r$, local disturbances $m$ and local primary control $u$. Moreover, the dynamics of extended state variables $\tilde{x}=\left[\begin{array}{ll}x & r\end{array}\right]$ depends on its own extended state variables comprising physical variables and the port variables [43], [42]; notably, the model structure holds for general multi-energy dynamics, see [44]. ${ }^{1}$ Shown in Figure 9 is also the mapping from the physical state space model into a higher-level model defining dynamics of aggregate state variable $x_{z}=\left[\begin{array}{lll}E & p & E_{t}\end{array}\right]$. It can be seen that this model is technology-agnostic and it is linear with respect to aggregate variables and it is directly affected by the rate of change of interaction variables $\dot{z}^{r, i n}$ entering the subsystem. In Figure 9 this aggregate model has two state variables and $E_{t}$ is considered to be a state dependent local disturbance. The energy in tangent space $E_{t}$ can also be modelled as an aggregate state variable [46]. The structure of the aggregate model in energy space is technology-agnostic and lends itself well to simulating dynamic interactions between components and to their systematic control, described next.

\section{A zoomed-out unified modelling of system-level dynamics using intVars}

A zoomed out model in energy space comprises a zoomed out model of each module, which upon interconnection is subject to general conservation laws in terms of intVars. Much the same way as the dynamical model of low-level zoomedin module, it has structure which states that the physical dynamics depends on the states and the dynamics of its port variables, displayed in the low row of Figure 9. The dynamics

\footnotetext{
${ }^{1}$ In this paper only lumped parameter models are explicitly described; future work is needed to extend this structural modelling to multi-layered PDE modelling in which intVars are waves [45].
}

of aggregate state variables of a zoomed out subsystem model can be shown to depend on its own aggregate variables and on the rate of change of interaction variables created by the neighbouring subsystems. To show this, observe that the interaction variables defined in Eqn. (2b) can be re-written as a dynamical model in terms of energy variables as shown in Eqn. (3). Here, the state variables are aggregate dynamical energy variables denoted as $x_{z, i}=\left[E_{i}, p_{i}\right]^{T}$.

$$
\begin{aligned}
& \text { Energy space state dynamics: } \quad x_{z, i}(0)=x_{z, i 0} \\
& \dot{x}_{z, i}=A_{z, i} x_{z, i}+B_{t} E_{t, i}\left(\dot{x}_{i}\right)+B_{z}\left(\dot{z}_{i}^{r, o u t}+\dot{z}_{i}^{u}+\dot{z}_{i}^{m}\right) \\
& \text { Rate of change of common outputs: } \quad z_{i}^{r, \text { out }}(0)=z_{i 0}^{r, \text { out }} \\
& \dot{z}_{i}^{r, \text { out }}=\phi_{z, i}\left(x_{i}, r_{i}, u_{i}, m_{i}, \dot{z}_{i}^{r, \text { in }}\right)
\end{aligned}
$$

The energy state space evolution is given in Eqn. (3a), which was first postulated in [39] as a generalization of first and second laws of energy conservation principles. The quantity $E_{t, i}$ is the stored energy in tangent space [39]. This variable is a quadratic function of local state variable derivatives, thereby representing higher-order effects, which is treated as an additional bounded disturbance by the energy space model to preserve the linearity in terms of the energy state variables. In Eqn. (3), the constant matrices and vectors used in the model are: $B_{t}=[0,4]^{T}, B_{z}=\left[\begin{array}{ll}1 & -1\end{array}\right]^{T}$ for any component and matrix $A_{z, i}=\left[\begin{array}{cc}0 & -1 / \tau_{i} \\ 0 & 0\end{array}\right]$ depends only on the time constant $\tau_{i}$. Each of the control and disturbance ports are associated with respective interactions, denoted using superscripts $u$ and $m$ respectively as follows: $\dot{z}_{i}^{u}=\left[P_{i}^{u}, \dot{Q}_{i}^{u}\right]^{T}$ and $\dot{z}_{i}^{m}=\left[P_{i}^{m}, \dot{Q}_{i}^{m}\right]^{T}$, respectively. These vector entities 
comprise instantaneous power and rate of change of generalized reactive power that can be defined using respective ports' effort and flow variables [39], [40].

\section{E. Interactive zoomed-out model in energy space}

We further consider the common output variable as the interaction variable defined in Eqn. (2b). This variable can only be computed numerically by utilising the analytical expressions given for the rate of change of interaction variable through an abstract map $\phi_{z, i}$ of internal variables as shown in Eqn. (3b) and depicted in Figure 9 [42]. Since outgoing interaction variable by definition is a function of local states and state derivatives (function of port input $r_{i}$ ), the rate of change of outgoing interaction variable depends on the rate of change of incoming interaction variable. Such dependence makes the modelling framework inherently interactive. For more details on the interactive modelling approach, the reader is referred to [39], [47], [42].

\section{EVOLUTION PATH FROM TODAY'S HIERARCHICAL CONTROL: EXAMPLES OF MULTI-LAYERED ENERGY MODELLING AND CONTROL}

This newly-introduced multi-layered modelling in energy space lends itself well to re-visiting many problems of direct interest to industry, their currently proposed solutions, underlying assumptions and to identifying possible enhancements. We illustrate several examples of using such interactive multilayered approach which have been introduced over the past 10-15 years in collaboration with many former students and colleagues.

\section{A. Available transfer capability (ATC), cross-border trading and seams}

The ATC-related seams and cross-border trading and operating problems reviewed in earlier Section II can be fully overcome by implementing interactive information exchange between the BAs for checking the feasibility conditions using the higher-level energy space model. The solution method proposed some time ago is effectively an implementation of this idea; it was shown in this work that exchange of tie-line flow information when scheduling resources in a multi-BA system should be coordinated; potential benefits for such coordination of tie-line flows generally results in major system-level savings [48]. Tie-line flow exchange scheduling which does not account for zoomed-in BA operating constraints is misleading because it is not physically implementable. In reference to Figure 6, BAs (NY, NE, IESO,PJM) can be characterised in terms of their projected feed-forward intVars over any time horizon of interest. Shown in Figure 4 are real and reactive power exchanges between these BAs as a function of ATC management practice. In Figure 4 a) the entire NPCC is considered as a single interconnection and scheduling/control optimization can be carried out in a fully coordinated manner by a Regional Transmission Organization (RTO), Fiigure 6, Scenario C. This ultimate benchmark requires major regulatory changes, it would be like Western Europe operated by a single EMS. There are many obstacles to doing this, and, instead ATC is computed in an entirely bilateral way by the BA, Figure 4, b); also Figure 6, Scenario A. Instead, an interactive approach is possible by which BAs propose their ranges of intVars, and minimal coordination is carried out by one of the iBAs using only the information about the intVar ranges and not requiring internal knowledge about the BAs. This aggregate zoomed out model is very low complexity compared to the full detained model in Scenario C. It is used to optimize settings for intVars within the ranges offered by the BAs, and the BAs re-do their own scheduling to meet the intVar command. This scenario B is an example of multilayered energy modelling use, Notably, it makes coordination over very large RTOs computable and scalable. Extremely important for enabling power delivery across BAs to support decarbonization is that improved Scenario B (interactive multilayered scheduling of both real and reactive power) results in much higher power transfers (black dots) than Scenario A (red dots) on the interface between NY and NE, for example. Scenario B is near-optimal when compared to the optimal benchmark Scenario C; notably, scenario $C$ is sub-optimal relative to improved scenario $\mathrm{B}$ which optimizes both real and reactive power interactions. These results were obtained using large scale systems and realistic generation/demand patterns [8], [48]. Similarly, sketched in Figure 5 are results of simulating portion of European system to determine how much wind power can be delivered to Bavaria from the Northwest Germany. Using publicly available data, we have shown major increases in this delivery when utilizing Scenario B-based dispatch [9], Figure 5.

\section{B. Imbalance control and markets for frequency regulation}

The increased penetration of intermittent resources has led to previously un-experienced inter-area frequency and power oscillations [23] and major efforts are under way to introduce new products, such as manual Fast Frequency Regulation (mFFR), automated (aFFR), and even emergency frequency regulation products. These problems are one of the major reasons for utilising HVDC technologies and directly control tie-line flows between the countries in Northern Europe. Notably, the line flow congestion can be created by either ATCbased energy trading and/or by sharing reserves for frequency regulation. At present it is quite challenging to design HVDC line control for eliminating these inter-area oscillations, a growing challenge [31].

Earlier research has shown that this problem of ensuring efficient frequency regulation and stabilisation in multi-BA systems can be posed using multi-layered modelling in energy space [49]. In this work intVars were defined under the realreactive power decoupling assumption and using linearised real power-frequency model of BAs. It is straightforward to show, in hindsight, that the modelling for enhanced Automatic Generation Control (AGC) proposed in that earlier work is a particular case of a more general modelling in coupled PQ multi-layered energy space proposed here. An important aspect of understanding the more general structure is being 
able to implement interactive AGC which supports participation of both: 1) internal DERs, demand response and other unconventional controllable equipment embedded within a BA and, ii) capable of on-line power balancing, as well as HVDC controllers of tie-line flows in between the BAs.

Relaxing implied temporal separation of frequency regulation and stabilisation: Continuous fluctuations of power outputs from the wind power plants and solar panels generally cause ever-changing small frequency fluctuations. This raises the key question concerning validity of temporal separation between: 1) frequency regulation using AGC that assumes fast primary dynamics stable; and 2) primary controllers stabilising very fast frequency fluctuations. Instead, one can use a continuous time domain model in energy state space. Shown in Figure 10 is a sketch of results from this work indicating that continuous solar power disturbances, for example, will create frequency deviations that are hard to decompose into slow quasi-static and fast dynamic fluctuations, since an equilibrium is actually never reached. For this, it is then necessary to model and control dynamic continuous intVar at the interfaces of BAs. Examples of such modeling and control can be found in [49], [50].

C. Stabilisation problems and ways forward in systems with inverter-based renewables (IBRs)

Consider next a system with fast persistent disturbances, such as solar power or wind power generation, shown in Figure 10. As scheduling for energy balancing and frequency/voltage regulation is done, the primary control in conventional generation (governors, AVRs) and IBRs responds to the commands given by these higher levels so that the fast fluctuations get stabilised to these set points. Some newly emerging dynamical problems are caused by inadequate fast inverter controllers which switch at the high $(\mathrm{kHz})$ rate and result in electromagnetic (EM) oscillations of currents and voltages in inductors and across capacitors internal to iBRs. It was recently shown that the fast frequency deviations at that time scale are caused by the fast reactive power imbalances. Similarly, the voltage deviations are mainly caused by the fast real power imbalances. A two-level nonlinear controller can be designed to stabilise AC voltage across an RL load while the conventional IBR controller fails to do this [19].

\section{Enhancing transient stability by non-linear energy con- trollers of FACTS during faults}

Consider a small power system with two conventional generators and a thyristor control of series capacitors (TCSC) [51]. When a line is lost, resulting in a load loss, the generator with smaller inertia will begin to accelerate, and, unless the load is reconnected within the critical clearing time of this fast generator, the system will go out of synchronism. However, when an energy controller is used to control FACTS, the excess power from the generator is seen by the FACTS controller by sensing changes in its intVar and FACTS helps increase critical clearing time of the generator in danger of loosing synchronism. Shown in Figure 11 are fast intVars with and without energy controller [51]. This is probably the first proof-of-concept reported to demonstrate potential of very fast energy controllers for enhancing transient stabilisation.

\section{E. Enhancing transient stability by nonlinear energy con- trollers of SVCs and flywheels}

One of first limiting factors when determining how much intermittent power can be deployed is the problem of dealing with sudden large wind gusts, and/or sudden large changes in radiance. It is during these events that systems may experience inverter control-induced EM problems, such as the one reported in [21]. To illustrate such problems and potential of enhanced effects of energy controllers, we show on a small Flores Island the effect of short- and long-duration large wind gust, Figure 12. Static Var Compensator (SVC) does not have much storage but can transiently stabilise system response to sudden wind gust shown in Figure 12. However, for stabilising system response to longer-duration wind gust shown in Figure 12 , it is necessary to have an energy controller of storage, such as flywheel.

\section{SUMMARY: TOWARD IMPLEMENTABLE PROTOCOLS}

It should be evident from the examples discussed in the previous sections that future electricity services will rely on non-traditional controllable equipment and that the NERC regulatory standards which assume that these functions are only being performed by the conventional power plants must be further generalised to support seamless participation of many diverse stakeholders. A new architecture is required where control is exercised by multiple participants interacting through a standardised information exchange and within operating limits. We propose a set of principles for this distributed coordinated architecture that includes the properties of individual participants and the information to be exchanged so that the dynamic interactions of system components align the sub-objectives of grid participants with the technical requirements of the system and societal governance objectives. We recognise that industry is likely to view the proposed architecture as quite radical. In particular, letting go of fully centralised control is likely to raise major concerns by system operators. These concerns can only be addressed by systematically setting operating principles for provable performance. This too represents a major challenge since the power grid has never been designed for provable performance over wide ranges of operating conditions. Based on prior research we propose that today's hierarchical control can be extended to other controllable components through area control error (ACE) metrics-like those currently used for decentralised frequency regulation. Today, ACE measures only quasi-stationary cumulative energy deviations, and generalised metrics are needed to define and measure power and rate of change of power ranging from near-instantaneous fluctuations through cumulative energy deviations, referred to as the interaction variables (intVars). If every BA controls its intVars to within an acceptable threshold, the interconnected system comprising many BAs will balance energy and power at the right rates 

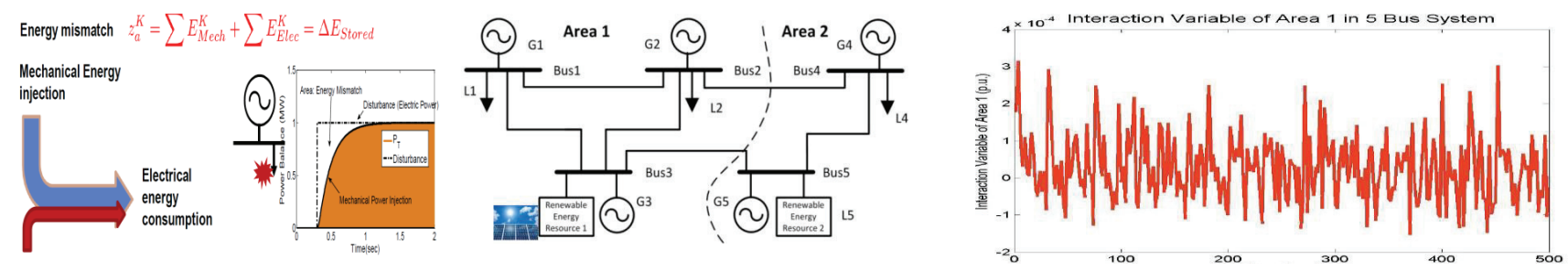

Fig. 10. Relaxing temporal regulation-stabilization assumption [49]

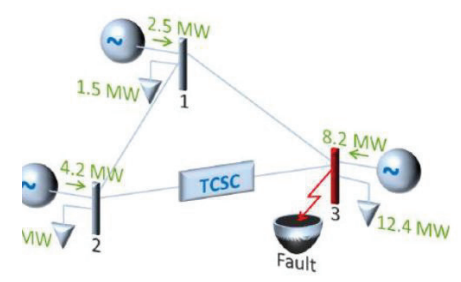

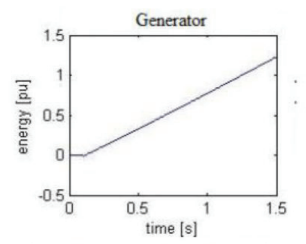
(a) Generator energy incre
uncontrolled system

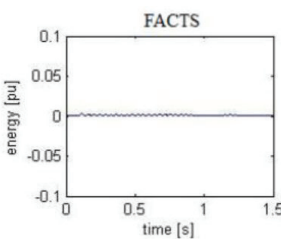

(b) FACTS energy increment in an uncontrolled system

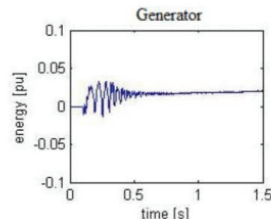

(c) Generator energy increment in a controlled system

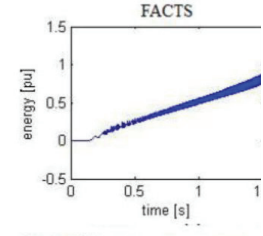

(d) FACTS energy increment in controlled system

Fig. 11. Increment of accumulated energy during fault [51]
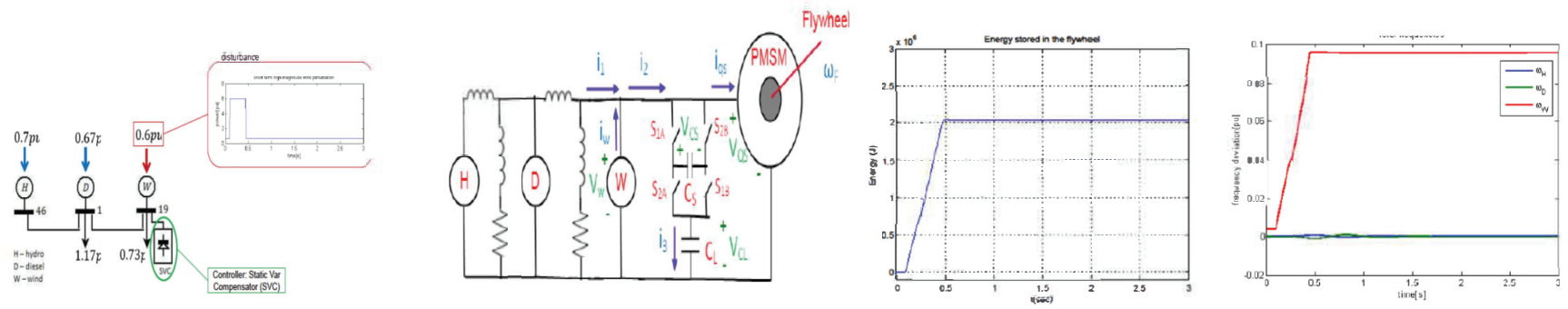

Fig. 12. SVC and flywheel energy control to stabilize system frequency during wind gusts [52]

and, not only will frequency will be regulated as it is today, but it will also be possible to implement sustainable and resilient operations and investment planning over multiple time scales. To exploit the potential of ACE for this purpose, it is necessary to break BAs into more granular iBAs - groups of stakeholders, distributed energy resources (DERs), and other end users who can proactively specify their abilities to generate/consume power and the rates at which they can do this. Creating iBAs with standardised intVar characteristics and minimally coordinating them within theoretically-set bounds makes it possible to manage currently overwhelming complex electricity system. When iBAs' physical untVar is characterised in terms of energy, power and rate of change of reactive power, the interconnected system will be feasible and stable with only relatively mild physically-intuitive conditions on iBAs' own responses and interactions. Since the decisions are internalised to all participants, system operators can rely on these intVar information instead of having to predict net system demand and pre-schedule generation. If the iBAs do not meet conditions for feasible and stable operations, the high-level coordinator can recommend adding fast storage. The spatial power delivery of the grid itself can be enhanced by controlling voltages and line reactances, but the power grid control needs to be re-engineered to accommodate this, e.g. by locating small DERs closer to the end users or installing re configurable circuit breakers. To summarise, we propose that the electrical grid architecture be re-conceived following three principles:

Principle 1: BAs transform to iBAs.: In order to support interactive control and co-design today's BAs are further organised as iBAs - groups of stakeholders, both utility and third parties, with their own sub-objectives. Each iBA is responsible for electricity services to its members and must communicate its commitments in terms of intVars to participate in electricity services with others.

Principle 2: Next generation SCADA to support this information exchange among iBAs.: As the operating conditions vary, stakeholders process the shared information, as sketched 
in Figure 2; optimise their own sub-objectives, subject to own constraints and preferences; and, communicate back their willingness to participate in system-wide integration.

Principle 3: The basic information exchange is in terms of energy, power and rate of change of reactive power intVars with physical interpretation as a generalised ACE.:

\section{ACKNOWLEDGEMENTS}

This paper is a result of financial support by the NSF project Early-Concept Grants for Exploratory Research (EAGER) "Fundamentals of Modelling and Control for the Evolving Electric Power System Architectures" project ECCS-2002570. This funding is greatly appreciated. Most important, the ideas put forward in this paper are outgrowth of many focused joined efforts with the author's graduate students at both Carnegie Mellon University and Massachusetts Institute of Technology.

\section{REFERENCES}

[1] Ilić, M. D., Jaddivada, R., \& Korpas, M. (2020). Interactive protocols for distributed energy resource management systems (DERMS). IET Generation, Transmission \& Distribution, 14(11), 2065-2081.

[2] Ilić, M. D. (2010). Dynamic monitoring and decision systems for enabling sustainable energy services. Proceedings of the IEEE, 99(1), 58-79.

[3] Gevorgian, V. and Booth, S., 2013. Review of PREPA technical requirements for interconnecting wind and solar generation (No. NREL/TP5D00-57089). National Renewable Energy Lab.(NREL), Golden, CO (United States).

[4] Ilić, Marija, Reynaldo Salcedo Ulerio, Edward Corbett, Eric Austin, Michael Shatz, and Erik Limpaecher. "A Framework for Evaluating Electric Power Grid Improvements in Puerto Rico." (2020).

[5] Ilić, Marija D., and Shell Liu. Hierarchical power systems control: its value in a changing industry. London: Springer, 1996.

[6] Allen, Eric H., Jeffrey H. Lang, and Marija D. Ilic. "A combined equivalenced-electric, economic, and market representation of the northeastern power coordinating council us electric power system." IEEE Transactions on Power Systems 23, no. 3 (2008): 896-907.

[7] Hogan, W. W. (1996). Markets in real electric networks require reactive prices. In Electricity transmission pricing and technology (pp. 143182). Springer, Dordrecht.

[8] Ilić. M., Lang, J., Cvijić, S., Allen, E., "Getting to 50 in 30", Presentation to NYISO, 2016.

[9] Ilić, M., Lang, J., Cvijić.,S., "New Electricity Transmission Software Solutions - The use of voltage dispatch to manage power transfers in Germany”, Duisburg, April 11, 2014 Workshop on Voltage/Var Management for Large-scale Integration of Renewable Energy Resources.

[10] Ilić, M., Cvijić, S., Lang, J.H. and Tong, J., 2015, July. Optimal voltage management for enhancing electricity market efficiency. In 2015 IEEE Power \& Energy Society General Meeting.

[11] Ilić, M., Lang, J., Litvinov, E., Luo, X. and Tong, J., 2011, December Toward coordinated-voltage-control-enabled HV smart grids. In 2011 2nd IEEE PES International Conference and Exhibition on Innovative Smart Grid Technologies.

[12] Ilic-Spong, M., Thorp, J. and Spong, M., 1986. Localized response performance of the decoupled Q-V network. IEEE transactions on circuits and systems, 33(3), pp.316-322.

[13] Cardell, J. B. (1997). Integrating small scale distributed generation into a deregulated market: control strategies and price feedback (Doctoral dissertation, Massachusetts Institute of Technology).

[14] Cardell, J. and Ilić, M., 2004, June. Maintaining stability with distributed generation in a restructured industry. In IEEE Power Engineering Society General Meeting, 2004. (pp. 2142-2149). IEEE.

[15] Holmberg, D., Burns, M., Bushby, S., McDermott, T., Tang, Y., Huang, Q., ... \& Neema, H. (2019). NIST Transactive EnergyModeling and Simulation Challenge Phase II Final Report. NIST special publication.

[16] Liu, Zhijian, and Marija D. Ilić. "Toward PMU-based robust automatic voltage control (AVC) and automatic flow control (AFC)." In IEEE PES General Meeting, 2010.
[17] Lu, Chao, Bonian Shi, Xiaochen Wu, and Hongbin Sun. "Advancing China? s smart grid: Phasor measurement units in a wide-area management system.” IEEE Power and Energy Magazine 13, no. 5 (2015): 60-71.

[18] Zaborszky, John, and Joseph W. Rittenhouse. "Electric power transmission; the power system in the steady state." (1954).

[19] Miao, X., \& Ilić, M. D. (2020). High Quality of Service in Future Electrical Energy Systems: A New Time-Domain Approach. IEEE Transactions on Sustainable Energy, 12(2), 1196-1205.

[20] Steinmetz, W.K. and Cole, J.D., 1993. Sampling ACE data for NERC CPC surveys. IEEE computer applications in power, 6(3), pp.27-32.

[21] Huang, F., and ERCOT Transmission Planning. "Panhandle and South Texas Stability and System Strength Assessment-Update." (2018).

[22] Anderson, P. M., Agrawal, B. L., \& Van Ness, J. E. (1999). Subsynchronous resonance in power systems (Vol. 9). John Wiley \& Sons.

[23] de Haan, Jerom ES, Carolina Escudero Concha, Madeleine Gibescu, Jan van Putten, Gerard L. Doorman, and Wil L. Kling. "Stabilising system frequency using HVDC between the Continental European, Nordic, and Great Britain systems." Sustainable Energy, Grids and Networks 5 (2016): 125-134.

[24] Ilić, M., Joo, J. Y., Carvalho, P. M., Ferreira, L. A., \& Almeida, B. (2013, August). Dynamic monitoring and decision systems (DYMONDS) framework for reliable and efficient congestion management in smart distribution grids. In 2013 IREP Symposium Bulk Power System Dynamics and Control-IX Optimization, Security and Control of the Emerging Power Grid.

[25] Ilić, M. D., Popli, N., Joo, J. Y., \& Hou, Y. (2011, July). A possible engineering and economic framework for implementing demand side participation in frequency regulation at value. In 2011 IEEE Power and Energy Society General Meeting (pp. 1-7). IEEE.

[26] Ilić, M., \& Jaddivada, R. (2019, September). Toward technically feasible and economically efficient integration of distributed energy resources. In 2019 57th Annual Allerton Conference on Communication, Control, and Computing (Allerton) (pp. 796-803). IEEE.

[27] Hammerstrom, D. J., Ambrosio, R., Carlon, T. A., DeSteese, J. G., Horst, G. R., Kajfasz, R., ... \& Thompson, S. E. (2008). Pacific northwest gridwise ${ }^{\mathrm{TM}}$ testbed demonstration projects; part I. Olympic peninsula project (No. PNNL-17167). Pacific Northwest National Lab.(PNNL), Richland, WA (United States).

[28] Salcedo, Reynaldo, Edward Corbett, Christopher Smith, Erik Limpaecher, Raajiv Rekha, John Nowocin, Georg Lauss et al. "Banshee distribution network benchmark and prototyping platform for hardwarein-the-loop integration of microgrid and device controllers." The Journal of Engineering 2019, no. 8 (2019): 5365-5373.

[29] Lin, Y., Eto, J. H., Johnson, B. B., Flicker, J. D., Lasseter, R. H., Villegas Pico, H. N., ... \& Ellis, A. (2020). Research roadmap on gridforming inverters (No. NREL/TP-5D00-73476). National Renewable Energy Lab.(NREL), Golden, CO (United States).

[30] Lasseter, R. H., Chen, Z., \& Pattabiraman, D. (2019). Grid-forming inverters: A critical asset for the power grid. IEEE Journal of Emerging and Selected Topics in Power Electronics, 8(2), 925-935.

[31] Halilbasić, L., Chatzivasileiadis, S. and Pinson, P., 2017, June. Coordinating flexibility under uncertainty in multi-area AC and DC grids. In 2017 IEEE Manchester PowerTech.

[32] Carvalho, P. M., Ferreira, L. A., Botas, J. C., Ilic, M. D., Miao, X., \& Bachovchin, K. D. (2016, June). Ultimate limits to the fully decentralized power inverter control in distribution grids. In 2016 Power Systems Computation Conference (PSCC).

[33] Castro, R., Almeida, M.E., Jesus, C., Carvalho, P.M. and Ferreira, L.A., 2012, April. Voltage Control Issues in Low Voltage Networks with Microgeneration. In SMARTGREENS (pp. 79-84).

[34] Willems, J. C. (2007). The behavioral approach to open and interconnected systems. IEEE control systems magazine, 27(6), 46-99.

[35] Happ, Harvey H. "Diakoptics-The solution of system problems by tearing." Proceedings of the IEEE 62, no. 7 (1974): 930-940.

[36] Uriarte, Fabian M. "On Kron's diakoptics." Electric power systems research 88 (2012): 146-150.

[37] Jaleeli, N., \& VanSlyck, L. S. (1999). NERC's new control performance standards. IEEE Transactions on Power Systems, 14(3), 1092-1099.

[38] M. Ilić and S.X. Liu. A simple structural approach to modeling and analysis of the inter-area dynamics of the large electric power systems: Part I- Linearized models of frequency dynamics, Proceedings of the 1993 North American Power Symposium, Washington, DC, pages 560 569, October 1993. 
[39] Ilić, Marija D., and Rupamathi Jaddivada. "Multi-layered interactive energy space modeling for near-optimal electrification of terrestrial, shipboard and aircraft systems." Annual Reviews in Control 45 (2018): 52-75.

[40] Wyatt, J. L., \& Ilić, M. (1990, May). Time-domain reactive power concepts for nonlinear, nonsinusoidal or nonperiodic networks. In IEEE international symposium on circuits and systems (pp. 387-390). IEEE.

[41] Penfield, P., Spence, R., \& Duinker, S. (1970). A generalized form of Tellegen's theorem. IEEE Transactions on Circuit Theory, 17(3), 302-305.

[42] Jaddivada, R., Ilić, M., A Feasible and Stable Distributed Interactive Control Design in Energy State Space, IEEE Conference on Decision and Control, December 2021.

[43] Ilić, Marija, Rupamathi Jaddivada, and Xia Miao. "Modeling and analysis methods for assessing stability of microgrids." IFAC-PapersOnLine 50, no. 1 (2017): 5448-5455.

[44] Bharadwaj, Pallavi, Janak Agrawal, Rupamathi Jaddivada, Min Zhang, and Marija Ilić. "Measurement-based Validation of Energy-Space Modelling in Multi-Energy Systems." In 2020 52nd North American Power Symposium (NAPS), 2021.

[45] Ilić, Marija D., and John Zaborszky. Dynamics and control of large electric power systems. New York: Wiley, 2000.

[46] Ilić, M., \& Jaddivada, R. (2020). Unified value-based feedback, optimization and risk management in complex electric energy systems. Optimization and Engineering, 1-57.

[47] Ilic, Marija D., and Rupamathi Jaddivada. "Fundamental modeling and conditions for realizable and efficient energy systems." In 2018 IEEE Conference on Decision and Control (CDC), pp. 5694-5701. IEEE, 2018.

[48] Ilić" "Corrective Resource Management for Voltage Support in Planning and Operation," FERC Staff Workshop on Voltage Coordination on High Voltage Grids Docket No. AD12-5-000, Washington DC, December 2011.

[49] Ilić, M. D., \& Liu, Q. (2012). Toward sensing, communications and control architectures for frequency regulation in systems with highly variable resources. In Control and Optimization Methods for Electric Smart Grids (pp. 3-33). Springer, New York, NY.

[50] Ilić, M., Xie, L., \& Liu, Q. (Eds.). (2013). Engineering IT-Enabled sustainable electricity services: The tale of two low-cost green azores islands (Vol. 30). Springer Science \& Business Media.

[51] Cvetković, M., \& Ilić, M. D. (2014). Ectropy-based nonlinear control of facts for transient stabilization. IEEE Transactions on Power Systems, 29(6), 3012-3020.

[52] Ilić, M., Cvetković, M., Bachovchin, K., \& Hsu, A. (2011, July). Toward a systems approach to power-electronically switched T\&D equipment at value. In 2011 IEEE Power and Energy Society General Meeting.

\section{Creative Commons Attribution License 4.0 (Attribution 4.0 International, CC BY 4.0)}

This article is published under the terms of the Creative Commons Attribution License 4.0

https://creativecommons.org/licenses/by/4.0/deed.en US 